Article

\title{
Wavelength Conversion Enhancement Achieved by Using Resonance in an Array of Nanocylinders
}

\author{
Kihwan Moon, Young Jin Lee, Seokhyeon Hong and Soon-Hong Kwon * \\ Department of Physics, Chung-Ang University, Seoul 06974, Korea; sinbadra@gmail.com (K.M.); \\ youngjin.lee.91@gmail.com (Y.J.L.); lechter@naver.com (S.H.) \\ * Correspondence: soonhong.kwon@gmail.com; Tel.: +82-2-820-5844
}

Received: 15 October 2017; Accepted: 27 November 2017; Published: 1 December 2017

\begin{abstract}
Upconversion (UC) materials are promising for harvesting visual light. However, the efficiency of UC processes is very low when applied to practical devices. Therefore, we propose an array of UC nanocylinders on a gold substrate and induce electric dipole (ED) and magnetic dipole (MD) resonances to increase the UC process efficiency by increasing light-matter interactions via the nanostructures. The nanocylinders not only increase the absorption of infrared light with a wavelength of $980 \mathrm{~nm}$ but also enhance the emission of visible light with a wavelength of $660 \mathrm{~nm}$ through surface plasmons and electric dipole resonances. The absorbance of the UC material can be enhanced by coupling with the surface plasmons and coupling with the MDs of each nanocylinder. On the other hand, the emission of visible light can be largely enhanced by increasing the spontaneous emission rate of the Purcell effect in electric dipole resonances and tailoring the output efficiency of the emitted light. In summary, we obtained an absorption enhancement of $\times 7.3$, an average effective upward emission enhancement of $\times 21$, and an improved total UC process of the proposed nanocylinder of $\times 155$.
\end{abstract}

Keywords: UC material; nanocylinder; Mie resonance

\section{Introduction}

Upconversion (UC) materials absorb light with low energy and emit converted light with higher energy [1,2]. For this, UC materials are have the potential to enlarge the finite spectral working range of the absorption band in practical devices, which is typically limited to visible light, so that it encompasses near infrared light. A number of devices based on UC processes have been studied for applications in solar cells [3-6], bio-imaging [7,8], and lasers [9,10]. However, the light absorbance in UC materials and the conversion efficiency of UC processes are usually too low for application in practical devices [11]. Therefore, several studies have combined metallic nanostructures with UC materials to improve the UC process in various ways [12,13]. For example, light absorption can be enhanced by applying localized surface plasmon (LSP) resonance to metal nanoparticles and nanowires $[13,14]$.

On the other hand, the dipole resonances due to Mie scattering can be introduced to increase light-matter interactions among subwavelength scale particles [15]. Electric dipole (ED) resonance can be induced not only in metal structures but also in dielectric structures. On the other hand, magnetic dipole (MD) resonance can be induced only in dielectric structures. Owing to the dipole resonances in dielectric structures, we can influence the direction of scattered light, enhance the interactions between light and particles (e.g., absorption), or enhance the emission of light depending on the resonant wavelengths. Indeed, various devices such as thin absorbers [16-19], lenses [20,21], and reflectors [22-24] have been reported that implement Mie resonances and appear in dielectric nanostructures.

In this paper, we introduced a nanocylinder composed of UC materials and a gold substrate to enhance the efficiency of the UC process. The absorption of $980 \mathrm{~nm}$ wavelength light or spontaneous 
emission in the UC nanocylinder with a wavelength of $660 \mathrm{~nm}$ can be enhanced through dipole resonances, surface plasmons, or electric hotspots due to coupling with the MD of each nanocylinder. In addition, we expect that the upward emission of the proposed structure is $\times 155$ larger than that of the reference structure when only the upward emission of visible light is collectable and meaningful.

\section{Upconversion Material Nanocylinder}

\subsection{Electric and Magnetic Dipole Resonances in a UC Nanocylinder}

First, we investigate the Mie resonances of a UC nanocylinder by calculating the scattering cross section using a three-dimensional (3D) finite-difference time-domain (FDTD) method. As shown in Figure $1 \mathrm{a}$, incident light with linear polarization along the $\mathrm{x}$-axis $\left(\mathrm{E}_{\mathrm{x}}\right.$-polarization) propagates along the z-axis and is scattered by a nanocylinder with height $h$ and a diameter $d$, in which the cylinder is made of the UC material. The UC material was assumed to absorb near-infrared light with a wavelength of $980 \mathrm{~nm}$ and emit visible light with a wavelength of $660 \mathrm{~nm}$. Because $\mathrm{Er}^{3+}$ doped $\mathrm{KNbO}_{3}$ has a higher upconversion efficiency at 530-550 $\mathrm{nm}$ (green) than at $660 \mathrm{~nm}$ (red), we focus on the red UC enhancement so that simultaneous efficient conversion of most of visible colors, green and red, from the NIR light can be realized. In addition, the mechanism to enhance the upconversion to red light by the resonances in an array of nanocylinder can be applied from the UC to green light in same way. For the nanocylinder with $h=400 \mathrm{~nm}$ and $d=400 \mathrm{~nm}$, the normalized scattering cross section ( $\left.\mathrm{Q}_{\text {scat }}\right)$, defined by the ratio between a scattering cross section and a geometrical cross section, is calculated in Figure $1 b$. First and second resonances are observed as peaks in the graph of $Q_{\text {scat }}$ at wavelengths of $710 \mathrm{~nm}$ and $960 \mathrm{~nm}$, which correspond to ED and MD, respectively. The electric field intensity profiles and vector plots for the two resonances in Figure 1c,d show that the resonances are ED and $\mathrm{MD}$, respectively. In particular, in the side view, the electric field of the ED near the middle of the cylinder is mainly oriented along the $x$-axis, which is similar to the $\mathrm{E}_{\mathrm{x}}$ dipole. In contrast, the electric field of the MD exhibits a current loop in the nanocylinder as the usual magnetic dipole.

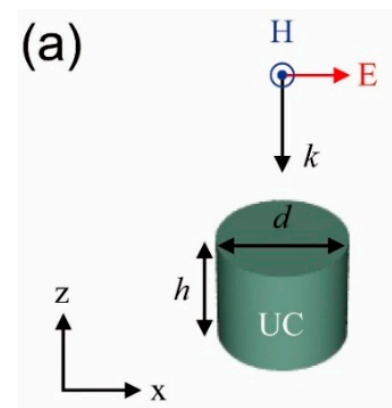

(c)

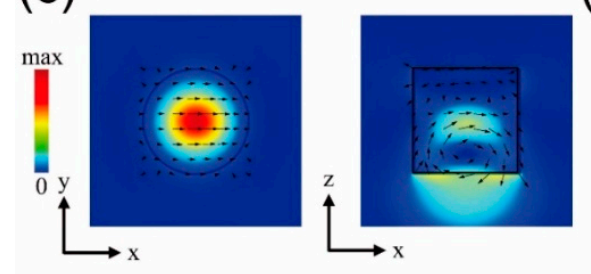

(b)

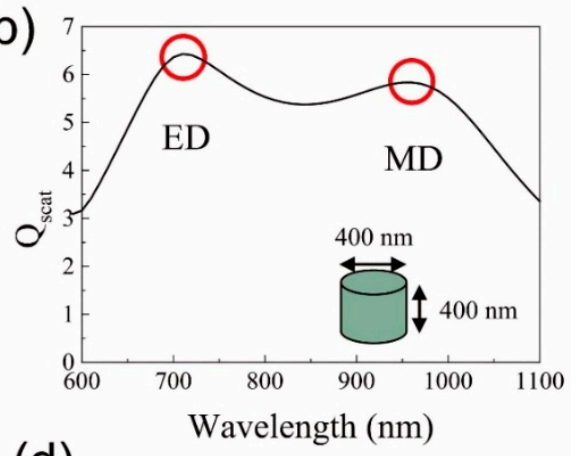

(d)

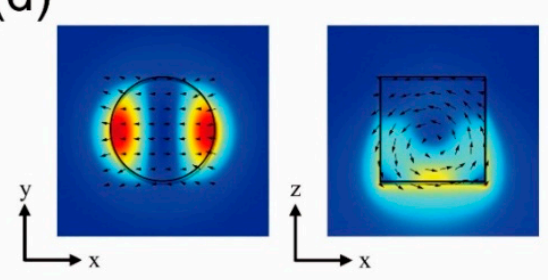

Figure 1. (a) A nanocylinder with height $h$ and diameter $d$ created using an upconversion material (UC). Incident light with a linearly polarized electric field along the $\mathrm{x}$-axis propagating along the $\mathrm{z}$-axis; (b) normalized scattering cross section ( $Q_{\text {scat }}$ ) of a UC nanocylinder with $h=400 \mathrm{~nm}$ and $d=400 \mathrm{~nm}$. Resonant wavelengths of the electric dipole (ED) and magnetic dipole (MD) are $710 \mathrm{~nm}$ and $960 \mathrm{~nm}$, respectively. Top and side views of the electric field intensity profiles and vector field distributions for (c) ED $(710 \mathrm{~nm})$ and $(\mathrm{d}) \mathrm{MD}(960 \mathrm{~nm})$. The top views are obtained from directly above the center of the nanocylinder. 
Here, we set the optical properties of the UC material and FDTD conditions as follows. In the simulation, we used $\mathrm{Er}^{3+}$-doped $\mathrm{KNbO}_{3}$ (with the refractive index plotted in the graph of Figure A1) as the UC material. This UC material absorbs $980 \mathrm{~nm}$ wavelength light and sequentially emits $660 \mathrm{~nm}$ [25-27]. The spatial grid of FDTD is $10 \mathrm{~nm}$ and the boundary conditions are perfectly matched layers in the single nanocylinder structure and a periodic boundary condition in the array structure. In addition, the gold is fitted as Drude critical points in the model [28].

\subsection{Tailoring the Wavelengths of ED and MD Resonances}

Resonant wavelengths of the Mie resonance in a nanoparticle depend on the geometry and the structure parameters. We calculated the normalized scattering cross section of a single UC nanocylinder to determine the dependences of ED- and MD-resonant wavelengths on the cylinder geometry, i.e., the height $(h)$ and diameter $(d)$, so that the emission and absorption wavelengths of the UC process match those of the ED and MD resonances. Figure 2 shows the $\mathrm{Q}_{\text {scat }}$ spectrum of single UC nanocylinders with different heights $(360 \mathrm{~nm}, 380 \mathrm{~nm}$, and $400 \mathrm{~nm}$ ) and a fixed diameter $(400 \mathrm{~nm})$, as well as for different diameters $(360 \mathrm{~nm}, 380 \mathrm{~nm}$, and $400 \mathrm{~nm})$ with a fixed height $(400 \mathrm{~nm})$. The resonant wavelengths of the ED and MD red-shift as the size of the nanocylinder, i.e., height or diameter, increases. However, the wavelength changes of ED and MD differ depending on if the diameter or height is varied. When the height changes from $360 \mathrm{~nm}$ to $400 \mathrm{~nm}$ by an amount of $40 \mathrm{~nm}$ while the diameter is fixed at $400 \mathrm{~nm}$, the ED wavelength increases by $40 \mathrm{~nm}$, and the wavelength shift of MD is larger at $50 \mathrm{~nm}$. In contrast, when the diameter increases from 360 to $400 \mathrm{~nm}$ with a fixed height of $400 \mathrm{~nm}$, the wavelengths of ED and MD increase $30 \mathrm{~nm}$ and $60 \mathrm{~nm}$, respectively. Using two different dependencies, the ED and MD resonances are allowed to match with the emission and absorption wavelengths, respectively, of the UC material.
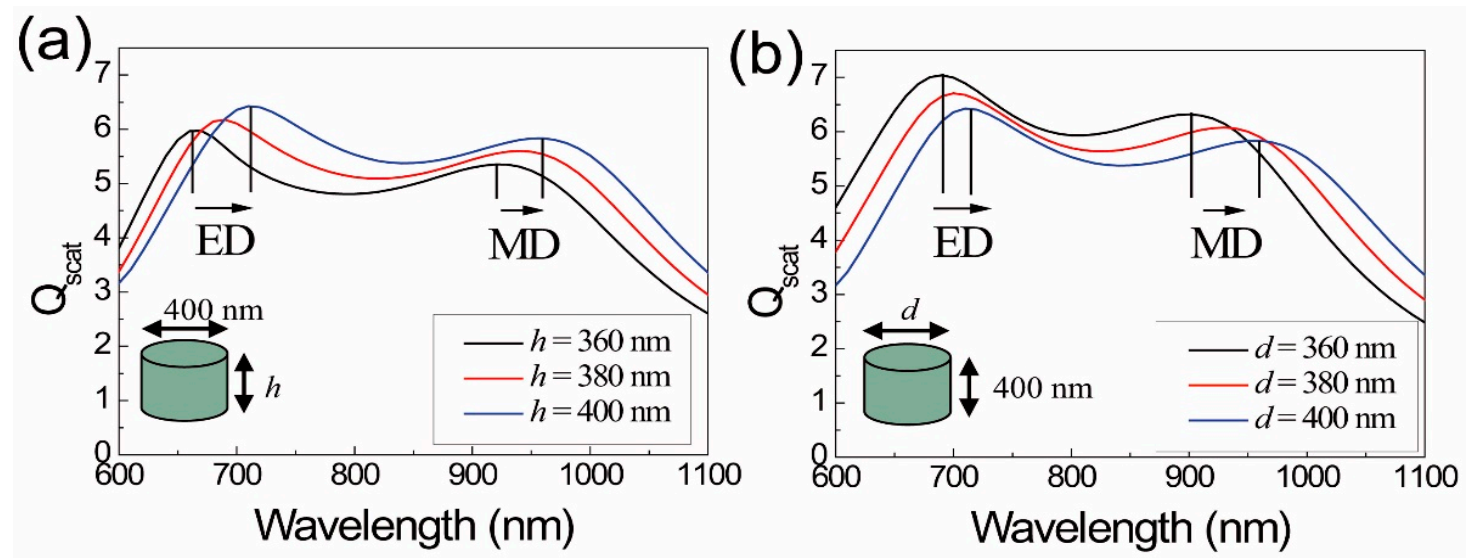

Figure 2. Normalized scattering cross section $\left(Q_{\text {scat }}\right)$ as a function of wavelength for a nanocylinder with (a) a fixed diameter (d) of $400 \mathrm{~nm}$ and different heights $(h)$ of $360 \mathrm{~nm}, 380 \mathrm{~nm}$, and $400 \mathrm{~nm}$; and (b) a fixed height $(h)$ of $400 \mathrm{~nm}$ and different diameters $(d)$ of $360 \mathrm{~nm}, 380 \mathrm{~nm}$, and $400 \mathrm{~nm}$. The resonant wavelengths of ED and MD increase with increasing $h$ and $d$.

\subsection{Periodic Structure of UC Nanocylinders}

To exploit a UC nanocylinder in a usable device, we consider an array of UC nanocylinders arranged in a square lattice with a period of $420 \mathrm{~nm}$, as shown in Figure 3a. The height and diameter of the nanocylinder are $400 \mathrm{~nm}$ and $400 \mathrm{~nm}$, respectively. We calculated the spectra for reflectance, transmittance, and absorbance in the nanocylinder array structure in Figure 3b. The absorbance has a peak of 0.56 at a wavelength of $730 \mathrm{~nm}$. In terms of the wavelength, the electric field vector flows of each nanocylinder are similar to those of the MD of a single nanocylinder (Figure 1d), and the overall vector flows for the entire surface of an array of nanocylinders is directed along the $x$-direction in phase, as shown in Figure 3c. Magnetic dipole resonance in each nanocylinder and the coupling 
between cylinders contribute to absorption enhancement. Because of the coupling between cylinders, a concentrated electric field, called an electric hotspot, is induced along the direction of the electric field of the incident lights. We are able to use this MD resonance of a single cylinder and the coupling effect of MDs in an array of nanocylinders by tuning the resonant wavelength of the resonance to the absorption wavelength of the material.

(a)

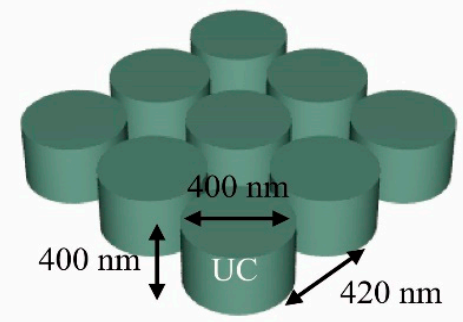

(c)

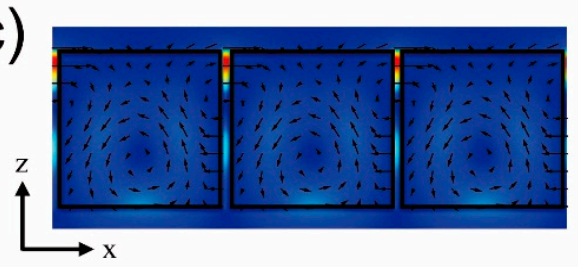

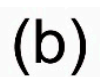

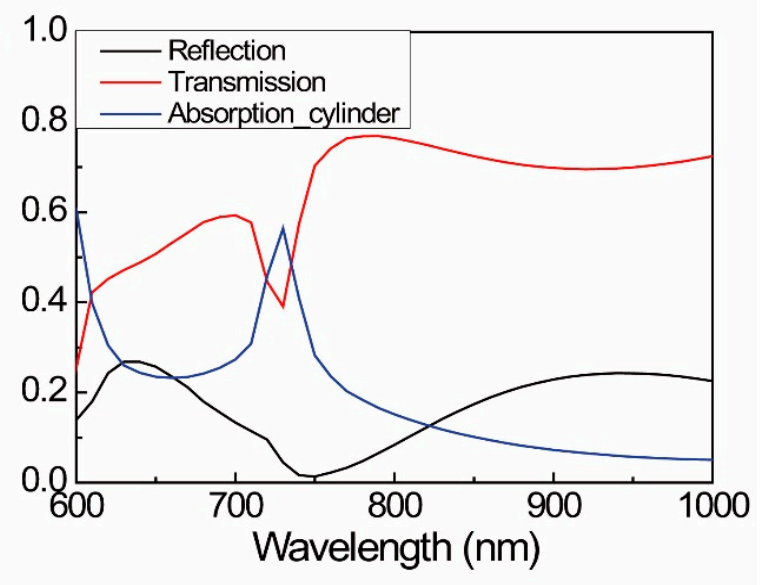

Figure 3. (a) Array of UC nanocylinders arranged in a square lattice with a period of $420 \mathrm{~nm}$. The cylinder has a height of $400 \mathrm{~nm}$ and a diameter of $400 \mathrm{~nm}$; (b) Reflection (black), transmission (red), and absorption curves (blue) as a function of wavelength; (c) Electric field intensity profile with a vector field plot in the side view.

\subsection{UC Nanocylinder on a Gold Substrate and an Absorption Enhancement at $980 \mathrm{~nm}$}

In order to increase the optical path length of the absorbed NIR (near-infrared) light and have uni-directional emission of the converted visible light, we introduce a gold substrate just below the array of nanocylinders, as shown in Figure 4a. The optimized structure has an absorption peak with a wavelength of $980 \mathrm{~nm}, h=300 \mathrm{~nm}, d=500 \mathrm{~nm}$, and a period of $520 \mathrm{~nm}$. In order to match the absorption peak of the nanocylinder array at the absorption wavelength of UC material, $980 \mathrm{~nm}$, we adjust the period to $520 \mathrm{~nm}$, because the peak wavelength depends on this period. As a reference structure, a thin UC film with a thickness of $130 \mathrm{~nm}$ on a gold substrate is assumed in Figure $4 \mathrm{~b}$, which has the same volume as the proposed structure. In the absorbance curve of Figure $4 c$, the reference structure is almost a constant value of $10 \%$ in the wavelength range of interest, $800 \mathrm{~nm}-1100 \mathrm{~nm}$. In contrast, the proposed nanocylinder structure has a sharp absorption peak of $980 \mathrm{~nm}$ with an absorbance of $67.1 \%$, which is 7.3 times larger than that $(9.2 \%)$ of the reference structure. According to the mode profile at $980 \mathrm{~nm}$, as shown in Figure 4d, a strong electric field intensity is observed at the bottom of the nanocylinder on a gold substrate and at the air slots between the nanocylinders. One field enhancement originates from the surface plasmon polaritons (SPPs), as indicated by the field intensity and the field direction normal to the gold substrate. The other enhancements are caused by the electric hotspots due to the coupling of MDs in the nanocylinders. The coupling of two resonances, SPP and electric hotspot, made a sharp absorption peak at $980 \mathrm{~nm}$, which is the absorption wavelength of the UC material. 
(a)

(b)

(c)
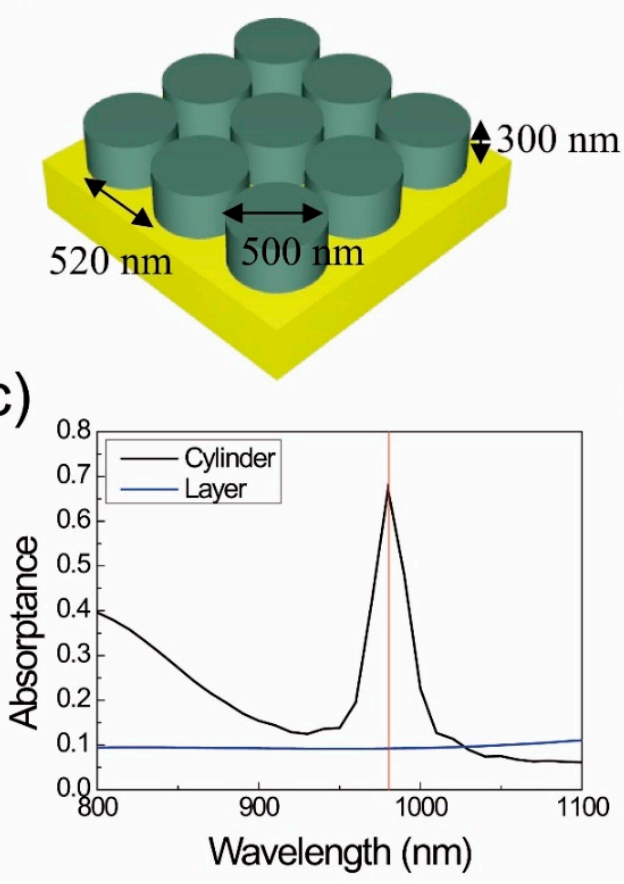

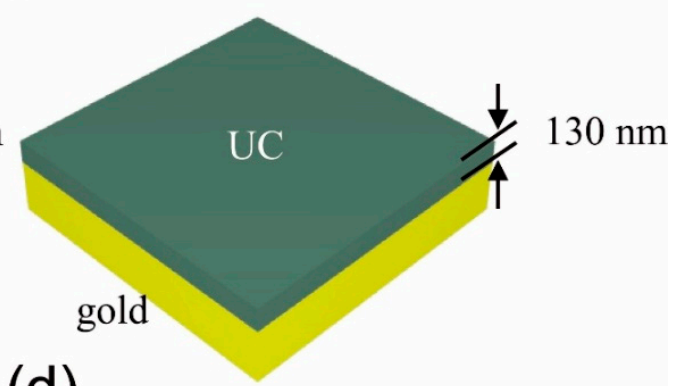

(d)

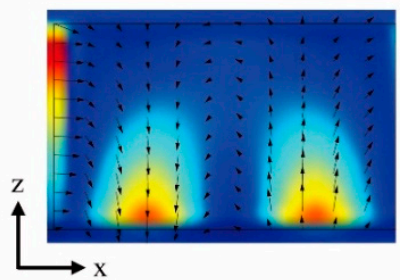

Figure 4. (a) Schematic of the proposed UC nanocylinder array structure with a gold substrate. Here, the UC cylinders have a height of $300 \mathrm{~nm}$ and a diameter of $500 \mathrm{~nm}$. The period in the array is $520 \mathrm{~nm}$; (b) Reference structure consisting of a UC film with a thickness of $130 \mathrm{~nm}$ and a gold substrate. The thickness is determined to have the same UC volume as the proposed nanocylinder array; (c) The absorptances of the proposed structure (black) and reference structure (blue) as functions of wavelength. The absorptance of a cylinder array is $67.1 \%$ at $980 \mathrm{~nm}$; (d) The electric field intensity profile with a vector field plot of the UC cylinder array.

\subsection{Spontaneous Emission Enhancement at $660 \mathrm{~nm}$}

Next, in order to investigate the emission properties of the converted visible light with wavelengths of $660 \mathrm{~nm}$, we calculated the spontaneous emission (SE) enhancement of dipole sources in the proposed structure, as seen in Figure $4 a$, which are horizontally polarized $\left(E_{x}\right)$ or perpendicularly polarized $\left(\mathrm{E}_{\mathrm{Z}}\right)$. SE enhancement is calculated using the following equation: SE enhancement $=($ emitted power of dipole source in UC nanocylinder)/(emitted power of dipole source in bulk UC material). We put a dipole source with a wavelength of $660 \mathrm{~nm}$ inside a UC nanocylinder and measured the total output power of the dipole source by integrating the Poynting vectors outgoing from the nanocylinder. We scanned the position of the dipole source with a spatial resolution of $20 \mathrm{~nm}$. The actual calculation region is set to a quarter of a nanocylinder due to the mirror symmetry of the nanocylinder.

The $E_{x}$ dipole source, parallel to the gold substrate, has a maximum SE rate enhancement of 2.0 at a plane with $\mathrm{z}=100 \mathrm{~nm}$ above the substrate, as shown in Figure $5 \mathrm{~b}$. Considering an image electric dipole on a gold substrate, which is anti-parallel to the original $E_{x}$ dipole source, the height of $100 \mathrm{~nm}$ corresponds to approximately a quarter wavelength of $660 \mathrm{~nm}$ in the UC material, such that the image dipole can constructively interfere with the original dipole [29]. The horizontal mapping of the enhancement is similar to the mode profile of the ED mode (left of Figure 1c), which indicates that the emission of the $E_{x}$ dipole source is enhanced via the Purcell effect of the ED mode in a nanocylinder [30]. The $\mathrm{E}_{\mathrm{y}}$ dipole source has the same spatial distribution as the SE enhancement of the $\mathrm{E}_{\mathrm{x}}$ dipole, except for a rotation by $90^{\circ}$. 
(a)

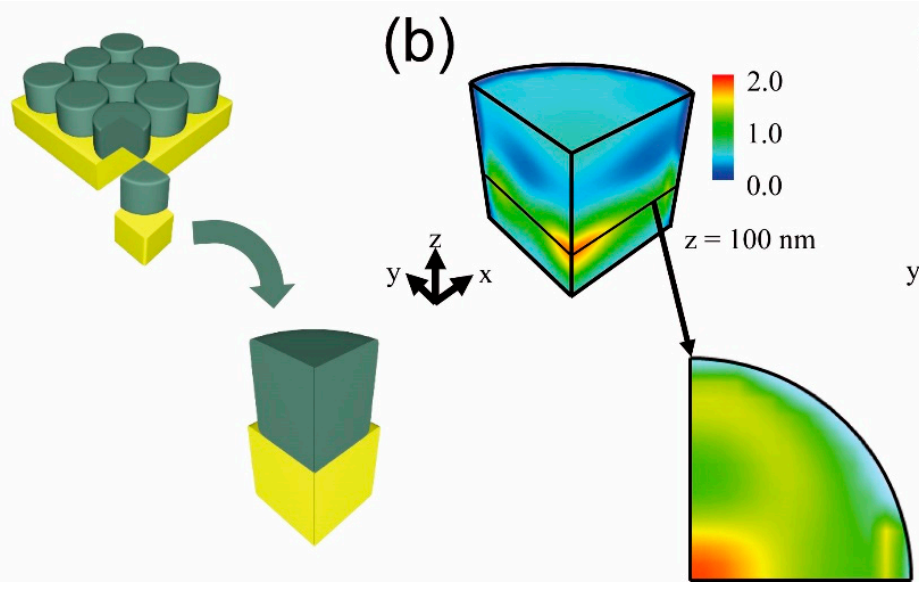

(c)

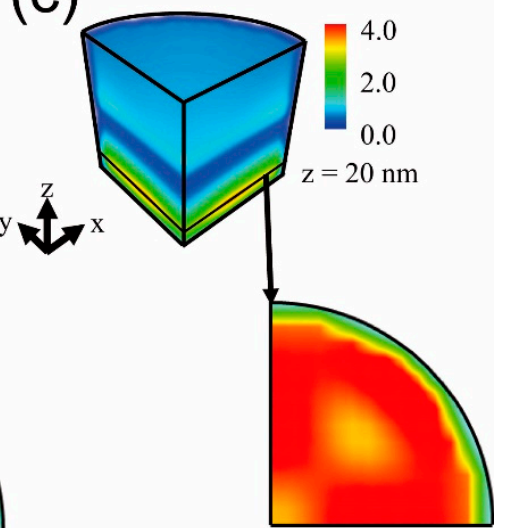

Figure 5. (a) A schematic diagram of one quarter of a UC nanocylinder to calculate the spontaneous emission (SE) enhancement mapping. The cylinder has a height of $300 \mathrm{~nm}$ and a diameter of $500 \mathrm{~nm}$. The period in the array is $520 \mathrm{~nm}$. SE enhancement distributions of (b) $E_{x}$ and (c) $E_{z}$ dipole sources; a magnified top view represents the horizontal SE enhancement mapping obtained at a plane with $\mathrm{z}=100 \mathrm{~nm}$ in the case of the $E_{x}$ dipole source and a plane with $\mathrm{z}=20 \mathrm{~nm}$ in the case of the $E_{z}$ dipole source, respectively. A plane with $z=0 \mathrm{~nm}$ represents the interface between the nanocylinder and gold substrate.

On the other hand, the $E_{z}$ dipole source emits light strongly at the surface of the gold substrate, which is shown in Figure 5c. Here, the maximum enhancement position $20 \mathrm{~nm}$ above the substrate is limited by the spatial resolution of the scanning $(20 \mathrm{~nm})$. If a smaller resolution is applied for the enhancement of the $E_{z}$ dipole, the maximum enhancement is at nearest position to the gold substrate. The SE rate of the $\mathrm{E}_{\mathrm{z}}$ dipole source is enhanced 4.0 times its normal maximum for the plasmonic mode. Finally, we summarize the SE rate enhancements at a visible wavelength of $660 \mathrm{~nm}$, the absorption enhancement at an NIR wavelength of $980 \mathrm{~nm}$ for the proposed nanocylinder array structure, and the reference structure in Table 1. The absorption of the nanocylinder structure is 7.3 times larger than that of the reference structure due to the fact it has coupled with the SPPs and the MDs in each nanocylinder.

For the emission of $660 \mathrm{~nm}$, the properties of the SE rate enhancements are very different depending on the direction of the dipole sources. In the case of parallel dipole sources $\left(E_{\|}\right)$such as $E_{x}$ or $E_{y}$ dipole sources, the $S E$ rate averaged over the nanocylinder is 0.62 times that of the SE rate in bulk UC material because the SE rate is suppressed near a half wavelength in the UC material [29], which is smaller than that of the reference structure. However, since the useful converted visible light is only the emitted light into free space, which escapes from the UC layer and propagates upward, an output efficiency, $\eta_{z}$, defined as the ratio between the upward-directional emitted power and the total emitter power, should also be considered alongside the SE rate enhancements. The output efficiency is same as the extraction efficiency in light-emitting diodes. The upward emission of the $660 \mathrm{~nm}$ wavelength light is $\times 0.66$ the total emitted power from the parallel dipole source in the nanocylinder structure due to constructive interference of the dipole source and the image dipole source on a gold substrate [29]; in contrast, the upward emission in the reference structure is only $\times 0.11$ of the total emitter power. Therefore, combined with the SE rate enhancements and the output efficiency, the effective upward emission of the parallel dipole source in the nanocylinder is 3.8 times larger than the reference structure. Here, the effective upward emission enhancement can be estimated by the ratio of the upward emission in the nanocylinder and the reference, in which the upward emitted power is obtained by the SE rate enhancement multiplied by the output efficiency, $\eta_{\mathrm{z}}$. On the other hand, in the case of a perpendicular dipole source $\left(\mathrm{E}_{\mathrm{z}}\right)$, the $\mathrm{SE}$ rate enhancements of the nanocylinder and the reference are similar, $\times 1.1$ and $\times 1.5$, respectively, because of the SPP coupling. However, the output efficiency of the nanocylinder $(0.53)$ is distinct from that of the reference $(0.01)$. In the reference that 
consists of a UC film on a gold substrate, the $E_{z}$ dipole source couples with a horizontally propagating SPP wave and the upward emission is strongly suppressed. In contrast, in the nanocylinder array, light from the $E_{z}$ dipole source is also able to couple to the SPPs, but the coupled SPPs can scatter at the edge of the finite nanocylinder, resulting in a high output efficiency of 0.53 . Therefore, the effective upward emission of the perpendicular source in the nanocylinder is 41 times larger than that of the reference due to its strong upward directionality. If two parallel polarized dipole sources, $E_{x}$ and $E_{y}$, and one perpendicularly polarized dipole source, $\mathrm{E}_{\mathrm{z}}$, are equally considered, the total effective upward emission of $660 \mathrm{~nm}$ light in the nanocylinder array is 21 times larger than the reference structure. As a result of the emission enhancement $(\times 21)$ and the absorption enhancement $(\times 7.3)$, the total effective enhancement of the UC process is $\times 155$ the reference structure due to SPP coupling, coupling between the MD mode, and effective vertical directionality.

Table 1. The volume average spontaneous emission (SE) enhancements of parallel $\left(\mathrm{E}_{\|}\right)$and perpendicular $\left(\mathrm{E}_{\mathrm{Z}}\right)$ dipole sources at a wavelength of $660 \mathrm{~nm}$ and the absorption enhancement at a wavelength of $980 \mathrm{~nm}$. The UC material absorbs near-infrared (NIR) light with a wavelength of $980 \mathrm{~nm}$ and emits visible light with a wavelength of $660 \mathrm{~nm}$. SE enhancements in an array of nanocylinders on a gold substrate and those in the reference structure are compared. SE enhancements in Table 1, the averaged SE enhancements, are calculated by integrating the values over the whole nanocylinder volume. The output efficiency $\eta_{z}$ is defined as the ratio between the upward-directional emitted power and the total emitter power.

\begin{tabular}{|c|c|c|c|c|c|c|}
\hline & \multicolumn{2}{|c|}{ Cylinder ( $h$ 300_d 500) } & \multicolumn{2}{|c|}{ Reference } & \multirow{2}{*}{$\frac{\text { Cylinder } z-\text { Emission }}{\text { Refernece } z-\text { Emission }}$} & \multirow{2}{*}{$\frac{\text { Cylinder Absoption }}{\text { Refernece Absoptio }}$} \\
\hline & SE Enhancement & $\eta_{z}$ & SE Enhancement & $\eta_{z}$ & & \\
\hline$E_{\|}$ & 0.62 & 0.66 & 1.0 & 0.11 & 3.8 & \multirow{2}{*}{7.3} \\
\hline$E_{z}$ & 1.1 & 0.53 & 1.5 & 0.01 & 41 & \\
\hline
\end{tabular}

\section{Conclusions}

In this paper, we proposed a dielectric nanostructure made of UC material that converts NIR light into visible light. The resonances in the structure enable the enlargment of the light-matter interactions in both absorption and emission to improve the efficiency of the UC process. The absorption at $980 \mathrm{~nm}$ is enhanced by an electric hotspot induced by the MD resonances and surface plasmons at the interface between the nanocylinders and a gold substrate. The absorption is 7.3 times larger than the reference UC film structure. In addition, we obtain the enhanced upward emission of $660 \mathrm{~nm}$ wavelength light by directional scattering of the emission light due to constructive interference and scattering of the nanocylinders. The upward emission rates are 21 times larger than those of the reference structure. In total, the efficiency of the wavelength conversion combined with the absorption and emission rates is improved by a factor of 155 . The converted light can be used effectively because the direction of the emitted light is upward. As a result, the efficiency of devices that use UC materials (such as solar cells and sensors) can be significantly improved.

$\mathrm{A} \mathrm{KNbO}_{3}$ thin film can be deposited on the silicon substrate using the polymeric precursor method (Pechini method) [31]. Gold thicker than several hundred nanometers is deposited on the $\mathrm{KNbO}_{3}$ film. Then, the sample is flipped and bonded to the other substrate. The original silicon substrate is removed by wet etching and the $\mathrm{KNbO}_{3}$ film on gold layer is obtained. The next process to form an array of nanocylinders can be performed by using conventional electron-beam lithography or nanostamp techniques.

Because the exciting electric field distribution in the absorption at $980 \mathrm{~nm}$ is spatially localized due to the resonance effects, as shown in Figure 4, the spontaneous emission enhancement can be affected by the spatial distribution of the absorption like in the case of lasing in nanocavity [32]. However, in order to consider the distribution adequately, there are several complicated factors to be considered such as the three-dimensional (3D) dynamics of carriers and photons in the multilevel system of a UC nanocylinder, carrier diffusion velocities, energy transfer rates in the UC process, 
and the 3D distribution of photon densities, which should be analyzed in a further study. Our analysis of the SE enhancements assumes that the $980 \mathrm{~nm}$ absorption generates the corresponding carriers in a nanocylinder uniformly, which can provide a reasonable value of the SE enhancements as long as the diffusion velocities are large enough compared to the size of a nanocylinder, $500 \mathrm{~nm}$.

Enhancements of the UC process by using nanostructures have been reported in other UC materials such as $\mathrm{ZnO} / \mathrm{Zn}$ [33] and InAs quantum dots (QDs) [34]. In $\mathrm{ZnO} / \mathrm{Zn}$, the nanostructure made by femtosecond laser ablation enhances the UC process from $800 \mathrm{~nm}$ to $500 \mathrm{~nm}$, with an enhancement factor of one order of magnitude. In InAs QDs, the flat bandedge property of a photonic crystal waveguide enhances the UC process, achieving lasing of $1.3 \mathrm{~mm}$ light from the absorption of $1.55 \mathrm{~mm}$ light. In contrast to such UC nanostructures that have small effective UC conversion areas of several micrometers, our UC nanocylinder array made by the lanthanide UC material can be fabricated on a wafer scale by nanostamp or nanosphere lithography techniques; thereby, the UC process increases by two orders of magnitude.

Acknowledgments: This work was supported in part by the National Research Foundation of Korea through the Korean Government under grant NRF-2013M3C1A3065051, NRF-2015R1A2A1A15055998, and NRF-2016R1C1B2007007.

Author Contributions: Kihwan Moon performed simulations and wrote the manuscript. Young Jin Lee and Seokhyeon Hong designed structures and performed simulations. Soon-Hong Kwon supervised all simulations and revised the manuscript.

Conflicts of Interest: The authors declare no conflict of interest.

\section{Appendix A}

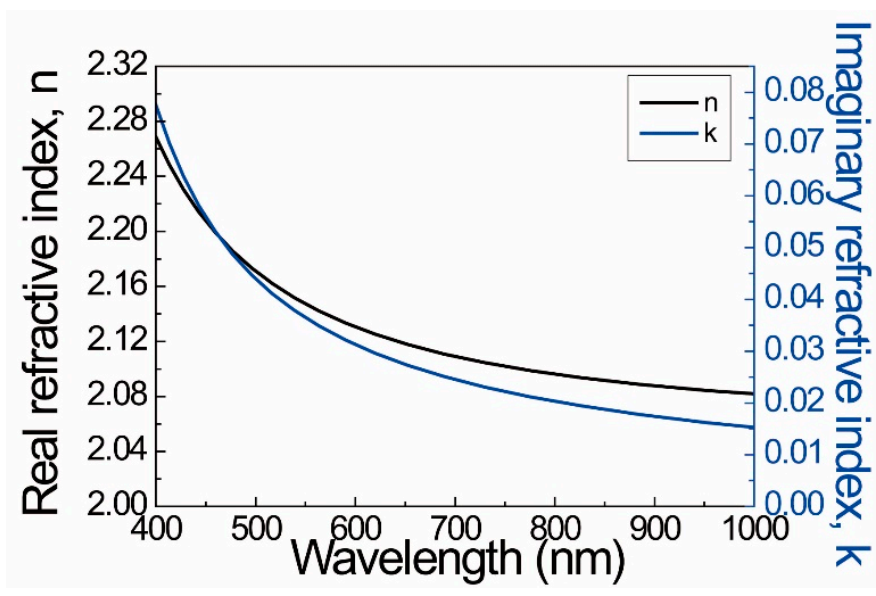

Figure A1. The experimentally measured refractive index (real: black; imaginary: blue) of the UC material, $\mathrm{Er}^{3+}$-doped $\mathrm{KNbO}_{3}$.

\section{References}

1. Menyuk, N.; Dwight, K.; Pierce, J.W. NaYF 4 : Yb,Er-An efficient upconversion phosphor. Appl. Phys. Lett. 1972, 21, 159. [CrossRef]

2. Yi, G.S.; Chow, G.M. Synthesis of hexagonal-phase $\mathrm{NaYF}_{4}: \mathrm{Yb}$,Er and NaYF4:Yb,Tm nanocrystals with efficient up-conversion fluorescence. Adv. Funct. Mater. 2006, 16, 2324-2329. [CrossRef]

3. Van Sark, W.G.J.H.M.; de Wold, J.; Rath, J.K.; Meijerink, A.; Schropp, R.E.I. Upconversion in solar cells. Nanoscale Res. Lett. 2013, 8, 81. [CrossRef] [PubMed]

4. $\quad$ Cheng, Y.Y.; Fuckel, B.; MacQueen, R.W.; Khoury, T.; Clady, R.G.C.R.; Schylze, T.F.; Ekins-Daukes, N.J.; Crossley, M.J.; Stannowski, B.; Lips, K.; et al. Improving the light-harvesting of amorphous silicon solar cells with photochemical upconversion. Energy Environ. Sci. 2012, 5, 6953-6959. [CrossRef] 
5. Ramachari, D.; Esparza, D.; Lopez-Luke, T.; Romero, V.H.; Perez-Mayen, L.; De la Rosa, E.; Jayasankar, C.K. Synthesis of co-doped $\mathrm{Yb}^{3+}-\mathrm{Er}^{3+}: \mathrm{ZrO}_{2}$ upconversion nanoparticles and their applications in enhanced photovoltaic properties of quantum dot sensitized solar cells. J. Alloys Compd. 2017, 698, 433-441. [CrossRef]

6. Yu, J.; Yang, Y.L.; Fan, R.Q.; Wang, P.; Dong, Y.W. Enhanced photovoltaic performance of dye-sensitized solar cells using a new photoelectrode material: Upconversion $\mathrm{YbF}_{3}-\mathrm{Ho} / \mathrm{TiO}_{2}$ nanoheterostructures. Nanoscale 2016, 8, 4173-4180. [CrossRef] [PubMed]

7. Nyk, M.; Kumar, R.; Ohulchanskyy, T.Y.; Bergey, E.J.; Prasad, P.N. High Contrast in Vitro and in Vivo Photoluminescence Bioimaging Using Near Infrared to Near Infrared Up-Conversion in $\mathrm{TM}^{3+}$ and $\mathrm{Yb}^{3+}$ Doped Fluoride. Nano Lett. 2008, 8, 3834-3838. [CrossRef] [PubMed]

8. Xiong, L.Q.; Chen, Z.G.; Yu, M.X.; Li, F.Y.; Liu, C.; Huang, C.H. Synthesis, characterization, and in vivo targeted imaging of amine-functionalized rare-earth up-converting nanophosphors. Biomaterials 2009, 30, 5592-5600. [CrossRef] [PubMed]

9. Lin, H.; Jiang, S.B.; Wu, J.F.; Song, F.; Peyghambarian, N.; Pun, E.Y.B. Er ${ }^{3+}$ doped $\mathrm{Na}_{2} \mathrm{O}_{-}-\mathrm{Nb}_{2} \mathrm{O}_{5}-\mathrm{TeO}_{2}$ glasses for optical waveguide laser and amplifier. J. Phys. D Appl. Phys. 2003, 36, 812-817. [CrossRef]

10. Singh, S.K.; Kumar, K.; Rai, S.B. Diode laser pumped $\mathrm{Gd}_{2} \mathrm{O}_{3}: \mathrm{Er}^{3+} / \mathrm{Yb}^{3+}$ phosphor as optical nano-heater. Appl. Phys. B 2010, 100, 443-446. [CrossRef]

11. Shalav, A.; Richards, B.S.; Trupke, T.; Kramer, K.W.; Gudel, H.U. Application of $\mathrm{NaYF}_{4}$ : $\mathrm{Er}^{3+}$ up-converting phosphors for enhanced near-infrared silicon solar cell response. Appl. Phys. Lett. 2005, 86, 013505. [CrossRef]

12. Lee, G.Y.; Jung, K.; Jang, H.S.; Kyhm, J.; Han, I.K.; Park, B.; Ju, H.; Kwon, S.J.; Ko, H. Upconversion luminescence enhancement in plasmonic architecture with random assembly of metal nanodomes. Nanocsale 2016, 8, 2071-2080. [CrossRef] [PubMed]

13. Park, K.; Jung, K.; Kwon, S.J.; Jang, H.S.; Byun, D.; Han, I.K.; Ko, H. Plasmonic nanowire-enhanced upconversion luminescence for anticounterfeit Devices. Adv. Funct. Mater. 2016, 26, 7836-7846. [CrossRef]

14. Guo, K.M.; Li, M.Y.; Fang, X.L.; Liu, X.L.; Zhu, Y.D.; Hu, Z.Q.; Zhao, X.Z. Enhancement of properties of dye-sensitized solar cells by surface plasmon resonance of Ag nanowire core-shell structure in $\mathrm{TiO}_{2}$ films. J. Mater. Chem. A 2013, 1, 7229-7234. [CrossRef]

15. Nam, M.; Kwon, N.-K.; Kwon, S.J.; Kwon, S.-H.; Cha, M.; Lee, S.-H.; Park, S.; Jeong, D.; Lee, K.-T.; Rhee, H.; et al. Multi-Functional Transparent Luminescent Configurationfor Advanced Photovoltaics. Adv. Energy Mater. 2016, 6, 1502404. [CrossRef]

16. Bezares, F.J.; Long, J.P.; Glembocki, O.J.; Guo, J.P.; Rendell, R.W.; Kasica, R.; Shirey, L.; Owrutsky, J.C.; Caldwell, J.D. Mie resonance-enhanced light absorption in periodic silicon nanopillar arrays. Opt. Express 2013, 21, 27587-27601. [CrossRef] [PubMed]

17. Liu, X.M.; Zhao, Q.; Lan, C.W.; Zhou, J. Isotropic Mie resonance-based metamaterial perfect absorber. Appl. Phys. Lett. 2013, 103, 031910. [CrossRef]

18. Callewaert, F.; Chen, S.; Butun, S.; Aydin, K. Narrow band absorber based on a dielectric nanodisk array on silver film. J. Opt. 2016, 18, 075006. [CrossRef]

19. Hong, S.; Lee, Y.J.; Moon, K.; Kwon, S.H. Double resonance perfect absorption in a dielectric nanoparticle array. Curr. Opt. Photonics 2017, 1, 228-232. [CrossRef]

20. Cheng, J.R.; Ansari-Oghol-Beig, D.; Mosallaei, H. Wave manipulation with designer dielectric metasurfaces. Opt. Lett. 2014, 39, 6285-6288. [CrossRef] [PubMed]

21. Gaillot, D.P.; Croenne, C.; Zhang, F.; Lippens, D. Transformation optics for the full dielectric electromagnetic cloak and metal-dielectric planar hyperlens. New J. Phys. 2008, 10, 115039. [CrossRef]

22. Moitra, P.; Slovick, B.A.; Li, W.; Kraychencko, I.I.; Briggs, D.P.; Krishnamurthy, S.; Valentine, J. Large-scale all-dielectric metamaterial perfect reflectors. ACS Photonics 2015, 2, 692-698. [CrossRef]

23. Moitra, P.; Slovick, B.A.; Yu, Z.G.; Krishnamurthy, S.; Valentine, J. Experimental demonstration of a broadband all-dielectric metamaterial perfect reflector. Appl. Phys. Lett. 2014, 104, 171102. [CrossRef]

24. Slovick, B.; Yu, Z.G.; Berding, M.; Krishnamurthy, S. Perfect dielectric-metamaterial reflector. Phys. Rev. B 2013, 88, 165116. [CrossRef]

25. Sun, S.Y.; Ge, Y.Y.; Zhao, Y.J.; Yuan, X.Y.; Zhao, Y.Z.; Zhou, H.P. Up-conversion luminescence behaviors in $\mathrm{Er}^{3+}$ doped single crystal $\mathrm{KNbO}_{3}$ nanosheets. RSC Adv. 2016, 6, 113038-113044. [CrossRef]

26. Balakrishnaiah, R.; Kim, D.W.; Yi, S.S.; Kim, K.D.; Kim, S.H.; Jang, K.W.; Lee, H.S.; Jeong, J.H. Frequency upconversion fluorescence studies of $\mathrm{Er}^{3+} / \mathrm{Yb}^{3+}$-codoped $\mathrm{KNbO}_{3}$ phosphors. Thin Solid Films 2009, 517, 4138-4142. [CrossRef] 
27. Wang, Y.L.; Wang, L.H.; Zhao, Y.J.; Yan, D. Improved luminescence behavior in $\mathrm{Er}^{3+}$ doped $\mathrm{KNbO}_{3}$ perovskite structure ceramics. Ceram. Int. 2016, 42, 17911-17915. [CrossRef]

28. Johnson, P.B.; Christy, R.W. Optical constants of the noble metals. Phys. Rev. B 1972, 6, 4370. [CrossRef]

29. Ee, H.S.; Kim, S.K.; Kwon, S.H.; Park, H.G. Design of polarization-selective light emitters using one-dimensional metal grating mirror. Opt. Express 2011, 19, 1609-1616. [CrossRef] [PubMed]

30. Purcell, E.M.; Torrey, H.C.; Pound, R.V. Resonance absorption by nuclear magnetic moments in a solid. Phys. Rev. 1946, 69, 37. [CrossRef]

31. Simoes, A.Z.; Ries, A.; Riccardi, C.S.; Gonzalez, A.H.; Zaghete, M.A.; Stojanovic, B.D.; Cilense, M.; Varela, J.A. Potassium niobate thin films prepared through polymeric precursor method. Mater. Lett. 2004, 58, 2537-2540. [CrossRef]

32. Nozaki, K.; Baba, T. Carrier and photon analyses of photonic microlasers by two-dimensional rate equations. IEEE J. Sel. Areas Commun. 2005, 23, 1411-1417. [CrossRef]

33. Li, H.; Zhang, C.-Y.; Li, X.-F.; Xiang, J.; Tie, S.-L.; Lan, S. Enhanced upconversion luminescence from ZnO/Zn hybrid nanostructures induced on a Zn foil by femtosecond laser ablation. Opt. Express 2015, 23, 30118-30126. [CrossRef] [PubMed]

34. Oda, H.; Yamanaka, A.; Ozaki, N.; Ikeda, N.; Sugimoto, Y. Operation of an InAs quantum-dot embedded GaAs photonic crystal slab waveguide laser by using two-photon pumping for photonics integrated circuits. AIP Adv. 2016, 6, 065215. [CrossRef]

(C) 2017 by the authors. Licensee MDPI, Basel, Switzerland. This article is an open access article distributed under the terms and conditions of the Creative Commons Attribution (CC BY) license (http://creativecommons.org/licenses/by/4.0/). 